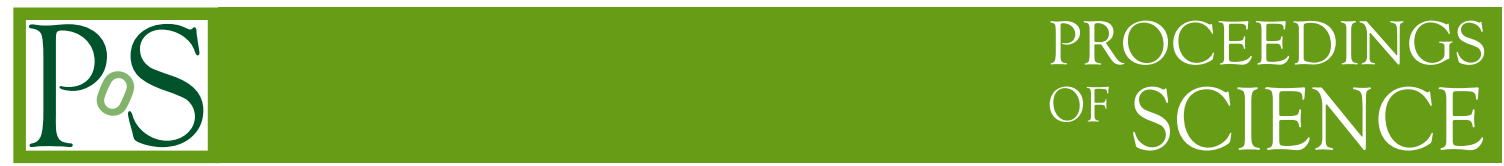

\title{
Jet charge modification in dense QCD matter
}

\author{
Haitao $\mathbf{L i}^{a}$ and Ivan Vitev ${ }^{b, *}$ \\ ${ }^{a, b}$ Los Alamos National Laboratory, \\ Mail Stop B283, Los Alamos, NM 87545, USA \\ E-mail: haitaoli@lanl.gov, ivitev@lanl.gov
}

\begin{abstract}
In these proceedings we report a recent calculation of the jet charge modification in heavy-ion relative to proton collisions at the LHC. Jets have played an essential role in constraining theories of in-medium parton shower evolution and in determining the properties of the quark-gluon plasma created in ultra-relativistic nuclear reactions. It is important to extend these studies to flavor-tagged jets and explore observables that are sensitive to their partonic origin. The average jet charge, introduced early on in the history of quantum chromodynamics, is a proxy for the electric charge of the quark or gluon that initiates the jet. In the framework of soft-collinear effective theory, we show how to evaluate the jet charge in a dense strongly-interacting matter environments. We identify observables that can isolate the contribution of in-medium branching from isospin effects and present predictions for the transverse momentum dependence of the jet charge distribution in nucleus-nucleus collisions and its modification relative to the proton case.
\end{abstract}

HardProbes 2020

1-6 June 2020

Austin, Texas

${ }^{*}$ Speaker 


\section{Introduction}

The jet charge is a substructure observable designed to approximate the electric charge of the hard scattered parton that initiates the jet. It was introduced in the late 1970s [1] and is defined as the transverse momentum-weighted sum of the charges of particles within the jet cone

$$
Q_{\kappa, \mathrm{jet}}=\frac{1}{\left(p_{T}^{\mathrm{jet}}\right)^{\kappa}} \sum_{\mathrm{i} \in \mathrm{jet}} Q_{i}\left(p_{T}^{i}\right)^{\kappa}
$$

Here, $Q_{i}$ and $p_{T}^{i}$ are the electric charge and the transverse momentum of particle $i$, and $\kappa>0$ is a free parameter. From the point of view of heavy-ion physics, the ability to identify the partonic origin of jets is extremely useful, as the modification in nuclear matter is different for quark and gluon jets [2]. Jet charge calculations for lead-lead $(\mathrm{Pb}+\mathrm{Pb})$ collisions at the LHC have been performed using a monte carlo approach [3] and the framework of soft-collinear effective theory (SCET) [4]. In these proceedings we review the latter. First measurements of the jet charge in heavy-ion collisions have also appeared and have been used to isolate the fraction of gluon-like jets [5].

Starting with the definition Eq. (1) and realizing that gluons do not contribute to the average jet charge, this observable can be expressed as follows:

$$
\left\langle Q_{\kappa, q}\right\rangle=\int d z z^{\kappa} \sum_{h} Q_{h} \frac{1}{\sigma_{\mathrm{q}-\mathrm{jet}}} \frac{d \sigma_{h \in \mathrm{q} \text {-jet }}}{d z}, \quad\left\langle Q_{\kappa, q}\right\rangle=\frac{\tilde{\mathcal{J}}_{q q}(E, R, \kappa, \mu)}{J_{q}(E, R, \mu)} \tilde{D}_{q}^{Q}(\kappa, \mu),
$$

where here $J_{q}(E, R, \mu)$ is a jet function. $\tilde{\mathcal{J}}_{q q}(E, R, \kappa, \mu)$ is the Wilson coefficient for matching the quark fragmenting jet function onto a quark fragmentation function and $\tilde{D}_{q}^{Q}(\kappa, \mu)$ is a fragmentation function [6]. The $(\kappa+1)$-th Mellin moments of the jet matching coefficient and fragmentation function are defined as

$$
\tilde{\mathcal{J}}_{q q}(E, R, \kappa, \mu)=\int_{0}^{1} d z z^{\kappa} \mathcal{J}_{q q}(E, R, z, \mu), \quad \tilde{D}_{q}^{Q}(\kappa, \mu)=\int_{0}^{1} d z z^{\kappa} \sum_{h} Q_{h} D_{q}^{h}(z, \mu) .
$$

In Eq. (3) $z=p_{T}^{i} / p_{T}, E$ is the jet energy, $R$ is the jet radius, and $\mu$ is the factorization scale. An important property of the jet charge is that it is sensitive to scaling violations in QCD

$$
\frac{p_{T}}{\left\langle Q_{\kappa, q}\right\rangle} \frac{d}{d p_{T}}\left\langle Q_{\kappa, q}\right\rangle=\frac{\alpha_{s}}{\pi} \tilde{P}_{q q}(\kappa),
$$

where $\tilde{P}_{q q}(\kappa)$ is the $(\kappa+1)$-th Mellin moment of the leading order splitting function. The effect has been measured in proton-proton collisions [7] and this serves as a strong motivation to extend the observable to heavy-ion collisions.

\section{Theoretical formalism in heavy ion collisions and numerical results}

Before we proceed to the evaluation of the jet charge in $\mathrm{Pb}+\mathrm{Pb}$ collisions we will validate the SCET formalism in the simpler $\mathrm{p}+\mathrm{p}$ reactions. The ATLAS collaboration has performed measurements of back-to-back jets at $\sqrt{s}=8 \mathrm{TeV}$, denoting them as a more forward and a more central jet, and extracted the flavor dependent jet charge

$$
\left\langle Q_{\kappa}^{f / c}\right\rangle=\left(f_{u}^{f / c}-f_{\bar{u}}^{f / c}\right)\left\langle Q_{\kappa}^{u}\right\rangle+\left(f_{d}^{f / c}-f_{\bar{d}}^{f / c}\right)\left\langle Q_{\kappa}^{d}\right\rangle .
$$



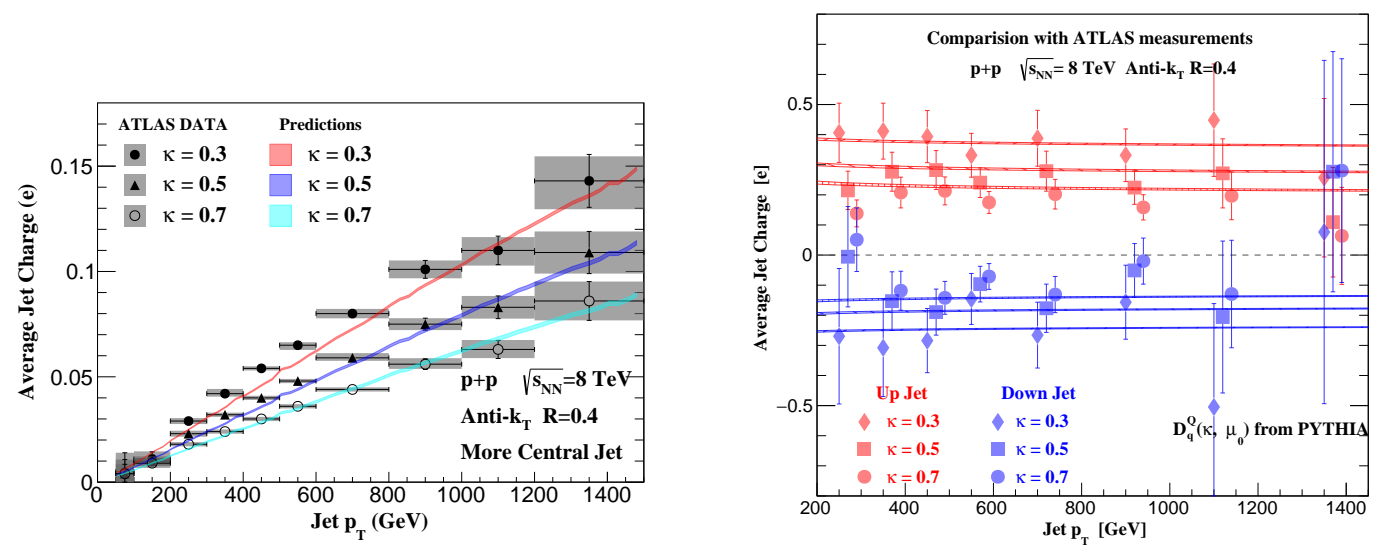

Figure 1: Left: transverse momentum dependence of the average jet charge distribution with $\kappa=0.3,0.5$ and 0.7 for the more central jets in $\sqrt{s_{\mathrm{NN}}}=8 \mathrm{TeV} \mathrm{p}+\mathrm{p}$ collisions at the LHC. Right: average charge of up and down-quark jets as a function of jet $p_{T}$. Data is from ATLAS [7].

In Eq. (5) $f_{q}^{f / c}$ is the fraction of $q$-flavored jets for the more forward/central jets and $\left\langle Q_{\kappa}^{q}\right\rangle$ is the average charge for the $q$ jet. Our theoretical results for the average jet charge and the up- and down-quark jet charges as a function of jet $p_{T}$ are shown in Fig. 1 . The average jet charge only relies on one non-perturbative parameter/boundary condition for a given $\kappa$ and the jet type, which we obtain through PYTHIA simulations. The uncertainties are evaluated by varying the factorization scale $\mu$ by a factor of two. The left panel of Fig. 1 gives the average jet charge for more central jets and its absolute value decreases with $\kappa$, as expected from Eq. (1). The right panel of Fig. 1 gives the flavor-separated charges for up- and down-quark jets. The predictions agree very well with the measurements by ATLAS [7], even though the data have large experimental uncertainties.

Propagation of partons in QCD matter adds a medium-induced component to the parton showers that characterize simpler reactions. The in-medium branching processes relevant to shower formation can be calculated order-by-order in powers of the mean number of scatterings [9]. An important characteristic of medium-induced showers, which persists to higher orders in $\alpha_{s}$ [8], is that they are softer and broader than the vacuum ones. Jet production and jet substructure in reactions with nuclei can be evaluated in a systematic and improvable fashion using a generalization of SCET to include interactions between its degrees of freedom and QCD matter mediated by Glauber gluons $\left(\mathrm{SCET}_{\mathrm{G}}\right)$. Thus, the ingredients of SCET factorization receive medium corrections where relevant. For example, QGP contribution to the the matching coefficients can be expressed in terms of the in-medium splitting kernels

$$
\mathcal{J}_{q q,(q g)}^{\mathrm{med}}(E, R, x, \mu)=\frac{\alpha_{s}(\mu)}{2 \pi^{2}} \int_{0}^{2 E x(1-x) \tan R / 2} \frac{d^{2} \mathbf{k}_{\perp}}{\mathbf{k}_{\perp}^{2}} P_{q \rightarrow q g,(g q)}^{\mathrm{med}}\left(x, \mathbf{k}_{\perp}\right) .
$$

The medium correction to the full quark jet function reads

$$
\begin{array}{r}
J_{q}^{\mathrm{med}}(E, R, \mu)=\int_{0}^{1} d x x\left(\mathcal{J}_{q q}^{\mathrm{med}}(E, R, x, \mu)+\mathcal{J}_{q g}^{\mathrm{med}}(E, R, x, \mu)\right) \\
=\frac{\alpha_{s}(\mu)}{2 \pi^{2}} \int_{0}^{1} d x \int_{0}^{2 E x(1-x) \tan R / 2} \frac{d^{2} \mathbf{k}_{\perp}}{\mathbf{k}_{\perp}^{2}} P_{q \rightarrow q g}^{\text {med,real }}\left(x, \mathbf{k}_{\perp}\right),
\end{array}
$$



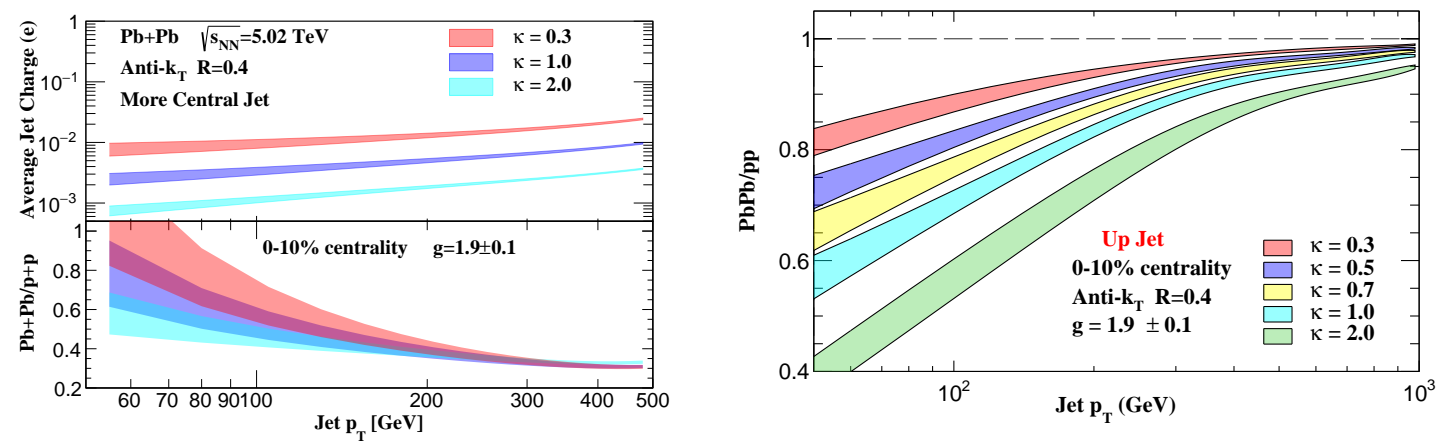

Figure 2: Left: The average jet charge in $\sqrt{s_{\mathrm{NN}}}=5.02 \mathrm{TeV}$ central $\mathrm{Pb}+\mathrm{Pb}$ collisions for more central jets and its modification relative to $\mathrm{p}+\mathrm{p}$ collisions. Calculations for $\kappa=0.3,1$ and 2 are shown. Right: Modification of the up-jet charge due to in-medium evolution as a function of transverse momentum.

see also [10]. Finally, in a QCD medium the evolution of the charge-weighted fragmentation function becomes

$$
\frac{d}{d \ln \mu} \tilde{D}_{q}^{Q, \text { full }}(\kappa, \mu)=\frac{\alpha_{s}(\mu)}{\pi}\left(\tilde{P}_{q q}(\kappa)+\tilde{P}_{q q}^{\text {med }}(\kappa, \mu)\right) \tilde{D}_{q}^{Q, \text { full }}(\kappa, \mu),
$$

where $\tilde{P}_{q q}^{\text {med }}(\kappa, \mu)$ is the $(\kappa+1)$-th Mellin moment of the medium splitting kernel. The additional scale dependence in the medium-induced part of Eq. (9) reflects the difference in the $k_{\perp}$ dependence of the vacuum and in-medium branching processes [11].

The jet charge and its modification in central $\mathrm{Pb}+\mathrm{Pb}$ collisions at the $\mathrm{LHC}$ are shown in the left panel of Fig. 2. At very high transverse momenta it is completely dominated by isospin effects. However, for $p_{T}<200 \mathrm{GeV}$ one begins to observe the effects of in-medium evolution. The uncertainty bands correspond to the variation of the coupling $g$ between the jet and the medium in the interval $(1.8,2.0)$. The need to cleanly isolate the contribution of in-medium evolution to jet charge modification led us to propose a new observable - the modification of individual flavor jet charge in heavy-ion versus proton collisions. This can be seen in the right panel of Fig. 2 where we show the medium modifications to the up-quark jet charge. The only difference between the up- and down-quark jet charges is the fragmentation function boundary condition, hence their modification is the same

$$
\frac{\left\langle Q_{\kappa, u}^{\mathrm{Pb}+\mathrm{Pb}}\left(p_{T}\right)\right\rangle}{\left\langle Q_{\kappa, u}^{\mathrm{p}+\mathrm{p}}\left(p_{T}\right)\right\rangle}=\frac{\left\langle Q_{\kappa, d}^{\mathrm{Pb}+\mathrm{Pb}}\left(p_{T}\right)\right\rangle}{\left\langle Q_{\kappa, d}^{\mathrm{p}+\mathrm{p}}\left(p_{T}\right)\right\rangle} .
$$

The individual jet charge modification eliminates the initial-state isospin effects and helps reveal the final-state medium-induced parton shower contribution to the jet function and the fragmentation function evolution. For this reason, the medium corrections are larger for smaller energy jets - a kinematic region where the medium-induced splitting functions are more important. Furthermore, when $\kappa$ is large the $(\kappa+1)$-th Mellin moment of the medium splitting function is more sensitive to soft-gluon emission. 


\section{Conclusions}

We presented recent calculation of the jet charge distributions in heavy-ion collisions in the $\mathrm{SCET}_{\mathrm{G}}$ effective field theory framework [4]. In the presence of nuclear matter the jet functions, jet matching coefficients, and the evolution of the fragmentation functions are constructed with the help of the medium-induced splitting kernels. The jet charge observable is particularly interesting because of its ability to discriminate between jets of various flavors, for example up-quark jets, downquark jets and gluon jets. This discriminating power remains valid in nucleus-nucleus collisions. Furthermore, the charge of jets can provide novel insight into the Mellin moments of mediuminduced splitting functions and the in-medium evolution of the non-perturbative fragmentation functions.

The jet charge definition is independent of the hard process. Thus, jet charge modification can be studied in other types of nuclear matter such as e+A collisions at the future electron-ion collider (EIC). Recent calculations of light and heavy meson production at the EIC have shown that with appropriate choice of center-of-mass energies and rapidity domains jet quenching effects in cold nuclear matter can be large and observable [12]. We plan to evaluate the jet charge in e+A reactions in the future.

\section{References}

[1] R. D. Field and R. P. Feynman, Nucl. Phys. B 136, 1 (1978)

[2] Y. T. Chien and I. Vitev, JHEP 05, 023 (2016) [arXiv:1509.07257 [hep-ph]].

[3] S. Y. Chen, B. W. Zhang and E. K. Wang, Chin. Phys. C 44, no.2, 024103 (2020) [arXiv:1908.01518 [nucl-th]].

[4] H. T. Li and I. Vitev, Phys. Rev. D 101, 076020 (2020) [arXiv:1908.06979 [hep-ph]].

[5] A. M. Sirunyan et al. [CMS], JHEP 07, 115 (2020) [arXiv:2004.00602 [hep-ex]].

[6] D. Krohn, M. D. Schwartz, T. Lin and W. J. Waalewijn, Phys. Rev. Lett. 110, no.21, 212001 (2013) [arXiv:1209.2421 [hep-ph]].

[7] G. Aad et al. [ATLAS], Phys. Rev. D 93, no.5, 052003 (2016) doi:10.1103/PhysRevD.93.052003 [arXiv:1509.05190 [hep-ex]].

[8] M. Fickinger, G. Ovanesyan and I. Vitev, JHEP 07, 059 (2013) [arXiv:1304.3497 [hep-ph]].

[9] M. D. Sievert, I. Vitev and B. Yoon, Phys. Lett. B 795, 502-510 (2019) [arXiv:1903.06170 [hep-ph]].

[10] Z. B. Kang, F. Ringer and I. Vitev, Phys. Lett. B 769, 242-248 (2017) [arXiv:1701.05839 [hep-ph]].

[11] Z. B. Kang, R. Lashof-Regas, G. Ovanesyan, P. Saad and I. Vitev, Phys. Rev. Lett. 114, no.9, 092002 (2015) [arXiv:1405.2612 [hep-ph]].

[12] H. T. Li, Z. L. Liu and I. Vitev, [arXiv:2007.10994 [hep-ph]]. 